POLIGRAFÍAS. REVISTA DE TEORÍA LITERARIA Y LITERATURA COMPARADA. NÚMERO 1. NUEVA ÉPOCA.

( ) UNAM TODOS LOS DERECHOS RESERVADOS

https://doi.org/10.22201/ffyl.poligrafiasnuevaepoca.2011.1.1654

\title{
El recurso de la ficción en la divulgación de la ciencia un asunto de alta costura. Reflexiones sobre El quinteto de Cambridge de John L. Casti
}

\author{
ANA MARÍA SÁNCHEZ MORA \\ Dirección General de Divulgación de la Ciencia, UNAM
}

The date is 1949, the venue is Christ's College, Cambridge, and guess who's coming to dinner? The guests are some of the greatest intellects to have twisted their minds around the problem of making machines that think. John Casti has invented an evening of spirited over-dinner argument to unveil the problems faced by machine intelligence". (John D. Barrow, citado en la cuarta de forros de The Cambridge Quintet; la cursiva es mía)

¿Cómo funciona la mente? ¿Cómo aprendemos, recordamos, vemos, oímos? ¿Qué es la conciencia? Aunque las dos primeras preguntas son sumamente complejas, muchos científicos esperan responderlas haciendo ingeniería inversa de nuestros circuitos neurales. Pero la conciencia, ese darnos cuenta de nosotros mismos, parece ser una incógnita que rebasa lo meramente físico; tan esotérica que durante la mayor parte del siglo XX la ciencia no la consideraba un tema de investigación; hoy es un problema de frontera. Una explicación de la conciencia sería un triunfo para las neurociencias.

Para el campo de la inteligencia artificial, el cerebro no es más que una máquina complicadísima cuyas propiedades pueden reproducirse mediante computadoras; que contiene muchas y distintas estructuras altamente especializadas que evolucionaron para resolver diferentes problemas. ¿Sería posible construir una computadora que tuviera una capacidad cognitiva semejante a la de un humano? ¿Podría desarrollarse una máquina a tal grado que le reconociéramos conciencia? Este tema de actualidad, que nos lleva a cuestionarnos qué hace tan especial al ser humano, no podía quedar fuera de la divulgación. Lo han abordado ya grandes divulgadores, y John L. Casti es uno de ellos. Pero independientemente del interés que despierta el tema de la inteligencia artificial, las características narrativas de su último libro 
(calificado por Ian Stewart, otro distinguido divulgador, como "un fascinante experimento literario") nos ofrecen una materia de reflexión muy provechosa a los interesados en las formas literarias de las que se vale la divulgación actual.

\section{La divulgación, ¿género literario?}

En la introducción a su obra de divulgación de la ciencia, La relatividad, Einstein asegura que, en aras de la claridad explicativa, no concede la menor atención a la elegancia de la presentación, en otras palabras, al estilo. "Me adherí escrupulosamente al precepto [...] según el cual el asunto de la elegancia debe dejarse al sastre [...]". Esta aclaración no pedida es digna de tomarse en cuenta porque nos hace notar que ya en 1916 flotaba en el aire la noción de que un texto de divulgación debía gozar de cierto estatus estilístico.

Pero, ¿desde cuándo se tuvo esa idea consciente de la necesidad de la buena escritura en las obras populares de ciencia? Carlos López Beltrán, reflexionando en torno al lenguaje y el conocimiento científico, dice:

Cualquier escrito científico del siglo dieciocho es por lo común un gozo. La voluntad de estilo, la cortesía y pulcritud con la que el lector es recibido en él, saltan a la vista. Rara vez hay un gesto arrogante o una fórmula enigmática gratuita que distancie al lector... Las relaciones entre indagar, experimentar, especular, calcular y escribir, eran fluidas, automáticas, incuestionadas [...] el avance del conocimiento empírico atado de modo indisoluble a la capacidad del lenguaje expresivo... Todavía ante un texto científico de hace cien años es común sentirse reaccionando: ¡pero qué bien escrito está! Lo que entre otras cosas lleva implícita la aceptación de que hay en el texto una aguda voluntad de comunicación, un esfuerzo consciente de expresarse bien [...] (López Beltrán, 2002: 231-232). ${ }^{1}$

Durante el siglo XX se acabó de producir la fractura entre la dificultad de la teoría y el lenguaje natural. Continúa López Beltrán:

La tecnociencia se fue diversificando [...] Las comunidades científicas comenzaron a insistir en que el lenguaje común, natural, resultaba torpe, estorboso, innecesario. Que si se quería conservar la verdad, la precisión, la eficacia de los hallazgos teóricos y experimentales, había que aislarlos en una densa capa de neohabla [...] Se acabó la buena escritura como un medio compartido [...] Ahora es una gracia, una especie de monería encontrar un investigador científico que escriba con estilo, que acierte a manejar con mínima destreza la lengua de todos para verter en ensayos o artículos

\footnotetext{
${ }^{1}$ Las cursivas que aparecen en las citas, a partir de este momento, son mías.
} 
no especializados sus cogitaciones, sus experiencias [...] Hoy que están vivos la mayoría de los investigadores científicos que han existido, muy pocos de ellos poseen control sobre la gramática, la retórica [...] para escribir de un modo no precodificado y abierto (López Beltrán, 2002: 232-233).

Las buenas cualidades, subrayadas por mí, reconocidas por la mayoría de los divulgadores y agradecidas por sus lectores, integran una gama que va desde la mera corrección lingüística hasta la capacidad del lenguaje expresivo que muchos ahora equiparan con lo literario, aunque ninguno ha podido definir sin lugar a dudas en qué consiste tal.

¿Cuándo calificamos a un texto de literario? Por supuesto, los criterios nunca son objetivos ni constantes, aunque en términos muy generales podemos decir que en la literatura intervienen la imaginación, un uso especial del lenguaje y la intención del autor. Esto podría referirse también, claro está, a los textos científicos. La diferencia residiría entonces en la serie de características en negritas, cuya consecución se basa en procedimientos subjetivos, azarosos y de recepción incierta. Aun así, es innegable que existen numerosos textos de divulgación ante los cuales la mayoría de los lectores exclama: “ipero qué bien escritos están!”.

Digamos que ese conjunto de textos conforman un género, es decir, obedecen a reglas específicas que permiten a los lectores reconocer no sólo su buena factura sino también la clase de texto que están leyendo. ¿Puede la divulgación de la ciencia ser considerada como un género literario, cuyas reglas podrían formularse explícitamente? Algunos analistas han intentado ya resolver esta cuestión.

Baudouin Jurdant (1993) parte de suponer que la divulgación es un género literario y se pregunta cómo puede reconocerse que un texto pertenece a tal género: es necesario extraer las reglas a las que obedece. Lo primero que propone es comparar la divulgación con otros tipos de obras literarias que tengan algún contenido científico, y encuentra que la ciencia ficción es la forma literaria más afín; tanto, que no puede identificarse ninguna diferencia formal entre ambas: comparten explicaciones, términos y datos, presentados como resultado de argumentos científicos; de modo que la diferencia entre esos dos tipos de textos debe residir en otra característica que no es el uso específico del lenguaje de la ciencia.

Jurdant hace notar que cuando uno comienza a leer ciencia ficción en forma de novela o de cuento, se le hace saber, a partir de claves integradas dentro del texto, que la lectura no está 
orientada hacia la adquisición de conocimiento: siempre habrá un héroe con quién identificarse; la mención de fechas transportará al lector a otra dimensión temporal; los eventos de la vida cotidiana lucirán diferentes debido a los aparatos usados o a algún otro truco como "tomó su píldora de desayuno y salió...", etc. "Sea cual sea la longitud de la narración", nos dice, "uno sabe del texto mismo que tiene un comienzo y, horas después, tendrá un final feliz o infeliz. La ciencia ficción está concebida para entretenimiento y placer literario" (Jurdant, 1993: 366).

Los textos de divulgación, en cambio, incluirán también claves formales, pero están diseñadas a propósito para poner al lector en una disposición de ánimo diferente: “La curiosidad del lector sentirá cosquilleos por la diferencia entre lo que se supone que sabe y lo que se supone que no sabe. Lo novedoso del contenido se hará sentir. El texto busca despertar la curiosidad; está construido para presentar conocimiento científico en definido contraste con sus ideas previas acerca del funcionamiento de las cosas" (Jurdant, 1993: 366).

En otras palabras, mientras que la ciencia ficción utiliza la ciencia para extrapolar un mundo posible (la descripción del cual hará que el lector reaccione diciendo: "plausible, pero no verdadero"), la divulgación usa la misma ciencia con el fin de provocar una reacción opuesta: "sin duda improbable, pero cierto", (ya que es la "ciencia genuina" la que lo dice).

La referencia a la verdad, lo que los divulgadores llamamos fidelidad a los conceptos científicos, parece ser esencial para describir apropiadamente la divulgación. Y es justamente esta pretensión de veracidad lo que da a este género literario su lugar propio y su estatus dentro del conjunto de la literatura, y no la forma literaria por sí misma. Pero esto, advierte Jurdant, nos deja con un grave problema. De acuerdo con algunos críticos literarios, la literatura tiene que ver con el parecido o la verosimilitud, no con la veracidad; pero si a la literatura no le concierne la verdad, ¿significa esto que la divulgación no es literatura?

Dicha suposición contradice el principio según el cual todo texto escrito es necesariamente parte de la literatura, pero va de acuerdo con la noción de que la forma literaria no es un componente esencial de la divulgación. Si, además, la pretensión de veracidad es incompatible con la literatura, y si los textos de divulgación quedan fuera de la crítica literaria pues sólo aparentan algo distinto a lo que son, ¿tenemos que abandonar la idea de que la 
divulgación es literatura? ¿Es realmente imposible descubrir las reglas implícitas que nos permitirían darle algún estatus dentro de la literatura?

Según Jurdant, hay otro género literario que nos permite una comparación más productiva: se trata de la "autobiografía genuina" (tipo confesiones), cuyas reglas están diseñadas para llenar las expectativas del lector haciendo referencia al género, es decir, el texto le advierte al lector sobre lo que va a leer, al igual que sucede con la divulgación. Lo que hace difícil encontrar reglas para ambos géneros es la ausencia de límites predeterminados, tanto de espacio como de forma, para administrar el interés o las expectativas del lector. Entonces hablamos no de reglas sino de estilo, es decir, el elemento personal o singular de la escritura literaria.

¿Cómo puede definirse dicho estilo para la divulgación? Propone Jurdant que el autor nos hace partícipes de su verdad al tiempo que combina el lenguaje ordinario con palabras técnicas. Los términos científicos dan a las narraciones su "sello de verdadero"; garantizan el origen del texto, su credibilidad como producto genuino de la actividad científica. También hay un "efecto de realidad basado en una poderosa entidad con vida propia llamada ciencia", efecto que se produce no para los legos, sino para los propios científicos. Al multiplicar las presentaciones posibles para dar a conocer los resultados científicos, los divulgadores comunican el sentimiento de que hablan de algo que puede tratarse desde diferentes ángulos sin que se cambie su definición. Al entrar las palabras de la ciencia en el reino del lenguaje ordinario, es decir, al ser confrontadas con públicos legos, la nueva realidad se vuelve parte de la vida cotidiana y cumple así una "función autorreferencial para la ciencia" (Jurdant, 1993: 369$371)$.

Tal vez ésta sea la razón, dice Jurdant, de que la divulgación no forme realmente parte de la literatura: el hecho de que no posea reglas que la hagan un género literario como cualquier otro. La forma escrita es una de las posibilidades que utiliza para comunicar las nuevas definiciones científicas de la realidad, pero esta forma no es, y no debe parecer, esencial: debe darle al lector la impresión de que la realidad contenida en su presentación no depende de la forma literaria (pues algo demasiado literario haría inverosímil la autoridad científica). La única 
forma de transmitir esta idea al escribir requiere de una especie de negación de la relevancia de lo que podría considerarse literario.

En resumen, es difícil que la divulgación sea considerada parte de la literatura porque debe negar que pertenece a ella. Sin esta negación, sus pretensiones de verdadera no serían plausibles y su referencia a la realidad se volvería vacía. Tal vez por ello sea más apropiado y menos problemático clasificar la divulgación como un estilo narrativo que, en muchos casos y paradójicamente, incorpora componentes de ficción.

\section{El recurso de la narrativa}

Se supone generalmente que quien hace divulgación se dirige sólo al público general y no a los científicos (que pueden ser sus colegas), y que realiza una actividad esencialmente diferente de la investigación científica. También se afirma que la divulgación no está relacionada con el desarrollo del conocimiento y que su pertinencia está restringida al público lego. Con esta visión, la divulgación se ubica en la periferia de la actividad (investigación) científica. Su tarea central es meramente traducir los conceptos "producidos en un contexto profesional y especializado, a un lenguaje que sea fácilmente comprensible, y transmitir esta información a un público masivo, como parte del esfuerzo de las relaciones públicas de la ciencia" (Curtis, 1994: 420), o bien de un reparto democrático del saber. En consecuencia, un problema ya clásico es saber qué tanto es posible hacer esa traducción y, estrechamente relacionado, cuál es la mejor forma para llevarla a cabo.

Lo anterior nos ayuda a entender por qué muchos de los divulgadores actuales escriben en forma de narrativa: se ha sugerido de un tiempo acá que quien no escriba así no será leído y que, aun si no se supone que la divulgación pertenece a la literatura, ésta se puede tomar como un modelo que permite un acercamiento más amable a los temas científicos. En este caso se considera a la narrativa simplemente como un recurso para despertar y sostener el interés de sus lectores legos, a los que se supone que les repele o por lo menos les aburre la ciencia. ${ }^{2}$

\footnotetext{
${ }^{2}$ Muchos recetarios y manuales aconsejan a los divulgadores utilizar ese estilo en beneficio de su público (ver, por ejemplo, Carey (1995) y Sánchez Mora (2000)). ¿Cómo, si no, van a apelar a la sensibilidad del lector? Sin embargo, pocos estudios han examinado de manera práctica si las narraciones funcionan como facilitadores o si el estilo "al grano", directo, prescindiendo de elementos literarios (narrativos), es poco atractivo para los lectores, como
} 
Sin embargo, ya se reconoce que el público de los divulgadores no es sólo el lego sino también sus colegas científicos, cumpliendo la "función autorreferencial" a la que se refiere Jurdant. Hay un continuo de géneros para la comunicación de la ciencia, y debemos admitir que la divulgación es también parte del discurso científico, y a un grado influyente, como advierte John Brockman (1996) en La tercera cultura. Ninguno de los géneros es neutro, ni epistemológica ni social ni profesionalmente; según Ron Curtis (1994), el estilo narrativo es muy utilizado en la divulgación porque tiene un objetivo extra: permite al divulgador hacer subrepticiamente juicios de valor sobre asuntos científicos mientras aparenta solamente describirlos.

Curtis examina un género común en los medios masivos: la narrativa en forma de historia de detectives, muy socorrida en la divulgación porque "proporciona un repertorio interpretativo y un espacio cognitivo donde se expresa y apoya tácitamente una versión muy específica del método científico". "La ciencia es baconiana, dicen estas historias, y progresa mediante el método de inducción por eliminación" (Curtis, 1994: 422). Sólo tendrá éxito en su investigación el científico que siga este método, mensaje dirigido no sólo al público lego. El héroe de estas historias es el incansable, estudioso, cooperador y decente detective científico cuya labor es motivada primariamente por la curiosidad; raras veces aparece el villano, el investigador agresivo, competitivo, individualista. "Según el 'mito baconiano', el científico que labora cooperativamente con sus colegas es el que triunfará en su empresa de develar los secretos de la naturaleza", como lo hace el detective triunfante de los relatos policiacos (Curtis, 1994: 423). Dicha estrategia narrativa, formal y estructuralmente análoga a dicho método, nos dice Curtis, “descansa en la presuposición fundamental del periodismo de ciencia contemporáneo: la idea de que el periodista no sólo debe contar una historia, sino una 'pequeña

se ha sugerido. ¿El estilo narrativo tiene efectos positivos en la comprensión y en la percepción de la importancia de los temas o es contraproducente, y en qué grado? Los pocos estudios de los estilos de escritura han producido resultados contradictorios. Algunos encuentran que el uso de narrativa no hace más creíbles a los contenidos; otros afirman que el estilo "al grano" es menos eficaz, o menos seductor. Jean Kelly y su equipo (2003) hicieron una prueba con lectores dándoles dos versiones del mismo contenido, una directa y otra como historia de detectives. El estilo narrativo fue calificado como "mejor" que el directo en la evaluación de las características de la historia, pero no en interés, precisión, amenidad o credibilidad. Aquiles Negrete (2003) y Negrete y Cecilia Lartigue (2004) han medido la eficacia de la forma narrativa literaria para comunicar información científica y encuentran que, si bien ésta es más atractiva y disfrutable que el texto escolar tradicional, a la larga la cantidad de información retenida es equivalente. Estos resultados pueden ser importantes para los divulgadores que suponen que están sacrificando la claridad o la importancia de los temas cuando adoptan un estilo narrativo, pero temen ahuyentar al lector si son directos. 
historia perfecta' con un principio, un medio y un final perfectamente marcados, en otras palabras, una tradicional narrativa de resolución. Para lograrlo se requiere un principio moral no ambiguo que pueda dotar a la secuencia de eventos de un final moralizador" (Curtis, 1994: 428). En suma: se trata de un relato normativo que conduce a una moraleja.

Aunque tal vez los divulgadores ni siquiera sean conscientes de ello, Curtis piensa que las historias normativas sí se verbalizan aunque no explícitamente; al insistir en que la ciencia debe ser una "pequeña historia perfecta", lo que hacen es igualar el proceder de la ciencia con esta forma literaria. Esto se debe a que las narraciones son "afirmaciones metafóricas que sugieren una relación de similitud entre los procesos narrados y el tipo de narración utilizada" (Curtis, 1994: 430). La narrativa tradicional (piénsese en un cuento clásico) tiene un principio, un medio y un final definidos, pero al principio cualquier cosa es posible, a la mitad hay cosas más probables y al final todo se sigue por necesidad. Dicho de otro modo, al transcurrir la narración las posibilidades se vuelven más limitadas y el final no parece una opción sino algo inevitable. A esto se le llama completez (redondez) o resolución autoevidente; al término del relato, ya no caben preguntas. Así procede la inducción por eliminación: un cierto número de hipótesis posibles que se van limitando a unas cuantas y al final del proceso "sólo queda una que se ve como necesaria o verdad autoevidente, el resultado inevitable de la investigación. Empieza con preguntas sin respuesta y termina con respuestas incuestionables"' (Curtis, 1994: 431).

Para Curtis, a menudo los divulgadores sólo pretenden ser inconscientes del acto mental mediante el cual organizan e interpretan los acontecimientos científicos utilizando concepciones literarias; fingen que su tema ya existía de alguna u otra manera en forma de relato, que simplemente se lo toparon y lo transmiten al lector. Esta práctica tiene un efecto importante: ya que los actos de interpretación y los entramados del divulgador no son notorios, tampoco lo son los juicios normativos o morales, que así no están expuestos a la crítica. La divulgación escrita en modo narrativo es un instrumento poderoso para promover una visión normativa y particular de la ciencia al tiempo que la hace inmune a la crítica. Es una forma de moralizar aparentando que sólo se describe. Por ello es que la narrativa es casi universal en la divulgación.

Curtis se pregunta si hay narrativas que puedan fortalecer a la divulgación al permitirle, al contrario de la narración baconiana, reflejar distintas interpretaciones críticas de la práctica 
científica. Propone que otras formas textuales pueden ser más apropiadas y padecer menos constricciones, de modo que logren transmitir concepciones diferentes de la racionalidad científica. Para desarrollar una nueva visión crítica de lo que es la ciencia, la divulgación necesita explorar otras formas literarias, en particular el diálogo socrático, radicalmente antibaconiano, porque finalmente, dice Curtis, en eso consiste la ciencia. En un diálogo de esta clase (o en una plática para intercambiar opiniones) no hay nada concluyente: la indagación científica empieza y termina con preguntas; no hay resoluciones. Los divulgadores suelen rehuir esta forma literaria que representa el "conflicto del pensamiento con el pensamiento" y, al hacerlo, muestran que las ideas baconianas predominan en la divulgación. Describir a los científicos enfrascados en debates, en lugar de logrando la unanimidad, es violar la "norma baconiana" (Curtis, 1994: 447). Los divulgadores dicen siempre y cada vez con mayor insistencia que el público necesita que se le hable más sobre el proceder de la ciencia, pero rara vez se le dice cuál es, excepto que la ciencia funciona por ensayo y error, algo que cualquier escolapio puede repetir.

Pero hay otra razón del escaso uso del diálogo: ésta es una especie de combate donde el lector es a la vez espectador y juez. Involucrar al lector es un peligro para quienes conciben a la divulgación como la encargada de relaciones públicas de la ciencia, que le transmite al público sólo la idea de acuerdos exentos de conflicto, ocultándole además que el "verdadero" conocimiento científico sólo es accesible a los propios científicos. Así se promueven el entusiasmo y el apoyo públicos, mas no la public understanding y mucho menos el partage du savoir. "El público puede ser un espectador, jamás un juez" (Curtis, 1994: 448).

Pero, ¿qué hay con el uso de la ficción en la narrativa de divulgación? ¿A dónde conduce la paradoja de incorporar elementos imaginarios a un discurso que, por definición, intenta transmitir una verdad, la verdad científica?

\section{Narrativa y ficción}

De lo antes expuesto, no puede negarse que el estilo narrativo en la divulgación es una poderosa herramienta tanto por su poder de atracción como por su naturaleza persuasiva. Pero la narrativa requiere necesariamente de elementos ficcionales para armar el relato y lograr esas 
dos cualidades. Si atendemos, por ejemplo, a la sugerencia de Curtis, es decir, utilizar el diálogo para mostrar de mejor manera el razonamiento científico, tendremos que construir una serie de personajes que sirvan de vehículo para lo que se discute, y un escenario donde transcurra el intercambio de ideas. En cuanto a lo que Jurdant propone, se hará el intento de darle al relato una forma literaria negando a la vez que se trata de literatura, con la finalidad de apuntalar la credibilidad del tema científico que se trata. El quinteto de Cambridge incorpora ambas propuestas y resulta por ello interesante analizar cómo lo lleva a cabo.

El argumento es, de manera muy sintética, el siguiente: es el año de 1949. El gobierno británico ha comisionado al científico y literato C.P. Snow para que indague entre la comunidad científica qué tan plausible es la construcción de una máquina que piense. Snow invita a cenar en sus aposentos de Cambridge a cuatro de los grandes intelectos que han abordado hasta entonces ese complejo problema teórico: J.B.S. Haldane, E. Schrödinger, A. Turing y L. Wittgenstein. En el transcurso de la velada, del aperitivo a los licores, los miembros del quinteto ofrecen sus puntos de vista sobre el apasionante y controvertido tema. El relato termina con la partida de los invitados.

Si estuviéramos interesados tan sólo en el tema de la inteligencia artificial, en los personajes históricos o en la fama de Casti como divulgador, nos dispondríamos a pasar un rato placentero, al tiempo que recibiríamos información científico-filosófica sobre un tema de actualidad. Demos por hecho lo anterior y coloquemos ahora el texto en la mesa de disección.

Para darle cuerpo al sencillo argumento, el autor sigue los pasos de la narrativa tradicional. Logra la ambientación mediante un escenario realista y palpable: un atardecer frío y lluvioso, la llegada en tren, las habitaciones en el Christ's College, la descripción prolija de platillos y bebidas. Se administra el interés del lector mediante el menú: la enumeración de los platos (a los que corresponden los títulos de los capítulos) nos da la sensación del transcurrir de la reunión; al mismo tiempo, las interrupciones propias de un acontecimiento social-culinario sirven de pausas para reactivar el interés y tomar un respiro que permita descansar de la ciencia: “The idea of a thinking machine is just too horrible to contémplate,' exclaimed Haldane, holding out his glass to allow Snow to pour him a little more of the Montrachet" (Casti, 1998: 70). “Stabbing at a piece of lettuce with his fork, Wittgenstein recalled somewhat 
sourly that this view of thought could be regarded as a kind of 'mentalese' coded into the brain" (Casti, 1998: 107).

La narrativa comienza cuando un nervioso Snow da indicaciones a su mayordomo (Simmons, personaje secundario pero utilísimo) sobre la puesta de la mesa; mientras tanto, los cuatro invitados, cada uno por su lado, están en tránsito hacia Cambridge. Los cinco personajes son descritos física y emocionalmente, y gracias a la inserción previa de una sección "Dramatis Personae" con las respectivas fotografías históricas (clásicas por reconocibles) y los datos biográficos, podemos imaginarlos claramente. Arriban intrigados por la sorpresiva invitación, Turing el último.

No es ninguna casualidad (aunque sí un golpe de suerte cronológico) que Casti haga coincidir en el espacio-tiempo del relato a cuatro representantes superlativos de otras tantas disciplinas: biología, física, computación y filosofía, mientras que el anfitrión representa la parte interdisciplinaria. Esto le permite abordar la inteligencia artificial de forma multifacética, facilitando el desarrollo de una discusión viva y sin los inconvenientes del monótono narradorpersonaje omnisciente. Los personajes no son estáticos: no obstante ser estrellas del firmamento intelectual, están dotados de la capacidad de modular sus puntos de vista de partida en respuesta al avance de la discusión. No hay héroes ni villanos: simplemente algunos nos resultan más simpáticos, al hacernos Casti partícipes de los pensamientos internos de los personajes:

Oblivious to the guard's disapproving glances, Turing spent most of the trip staring up at the ceiling, reflecting on the Lister address delivered earlier in the month by his Manchester University colleague, the famed neurosurgeon Sir Geoffrey Jefferson. How bloody wrong-headed can a man be, fumed Turing, to think that because a machine isn't built from biological parts like flesh and bones, and doesn't have emotions like a whimpering dog or a laughing baby, it's not capable of rational thought? Old Jefferson has really put his foot in it this time, Turing giggled to himself [...] (Casti, 1998: 10).

Para la mitad de la cena, todos los miembros del quinteto, que al principio pudieron parecernos distantes o excéntricos, se han humanizado, se han vuelto entrañables.

Sin siquiera haberlo planteado el anfitrión, sus huéspedes empiezan a hablar del tema por el que han sido requeridos. El fuego chisporrotea en la chimenea y la cena comienza. 
Conforme se sirven los platillos, los invitados discuten las diversas posturas, propias y ajenas, sobre la posibilidad de construir una máquina que piense como un humano. Se cuestionan sobre cuáles serían los principios científicos y filosóficos involucrados. La temática se construye mediante las participaciones enérgicas, viscerales o mesuradas, que permiten verter el conocimiento inacabado, los obstáculos epistémicos y cognitivos, las distintas versiones de las propuestas teóricas y sus contradicciones. Así, se llega entre todos al meollo del asunto: ¿qué es pensar?

El “diálogo socrático" se encarna, así, en el intercambio de opiniones. Casti no nos da un recuento secuencial, ni completo, ni definitivo (cosa que por otra parte sería imposible) de un tema científico. Lo presenta desde diferentes puntos de vista, algunos opuestos entre sí, ejemplificando lo que Curtis ha sugerido como una forma más cercana al método de la ciencia. Sólo la narrativa tiene un final cerrado. El relato termina cuando los invitados se despiden; Snow suspira, rellena su copa y se tumba en el sillón a pensar, con cierto aire desesperado, en cómo podría transmitir la diversidad de ideas que han circulado esa noche:

How can I possibly compare Wittgenstein's philosophical position against thinking machines with Turing's technical arguments? And where do Schrödinger's and Haldane's views on the origin of life and the way living organisms behave fit into this overall picture? As if this were not enough, Snow mused, there's also Wittgenstein's Hieroglyphic Room thought experiment. As he laid it out, it certainly appeared to be a convincing refutation of the notion that a machine could ever think like a man. Somehow, though, the force of that argument was weakened by the discussion of thought and language that followed. How can I begin to convey the subtleties of these conflicting themes and views to someone like the Minister, who takes his science in small bites and with no rough edges? Well, he concluded, polishing off the last of the cognac, I've written reports before with a lot less information to draw upon this. He got up from the sofa, stretched leisurely and then put out the lights and headed for the bedroom. The thinkingmachine conundrum isn't going to be resolved in one evening, he thought, and tomorrow is another day... (Casti, 1998: 171).

Los puntos suspensivos en el texto de Casti son la seña clara de que se ha terminado la narración, no así la discusión. Con este final abierto, el lector no puede menos que identificarse con Snow, algo así como: "no es posible concluir algo definitivo de todo esto tan interesante, pero ahora tengo más información y me doy cuenta de que la forma científica de razonar no es un camino lineal e infalible". 
No dudaríamos en clasificar El quinteto de Cambridge de entrada como novela, y al igual que en una novela histórica, la de Casti transcurre en un lugar y un tiempo ubicables en la realidad; también los personajes han existido y no sólo eso, han sido objeto de escrutinio histórico-científico por muchos autores. Sin embargo, aun cuando habría sido temporal y espacialmente posible, en realidad la reunión jamás se llevó a cabo. Nuestra disección se centra ahora en una compleja yuxtaposición: el contenido científico y las situaciones ficticias.

\section{¿Cómo distinguir ficción de realidad?}

La respuesta a esta pregunta indudablemente forma parte de las preocupaciones de Casti el divulgador. Consciente de que se ha acercado tanto como es posible a la literatura, se deslinda de ella; primero, añadiendo un subtítulo que deje bien claro de qué se trata: The Cambridge Quintet. A Work of Scientific Speculation; en seguida, mediante una declaración titulada

“Author's Note" al principio del libro, de modo que no pueda pasarle inadvertida al lector:

The book you hold in your hand is not a novel; but it is a work of fiction, part of an emerging genre that I like to label 'scientific fiction'. The Japanese term for this kind of work is a shosetsu, a rather more flexible an generous term than 'novel'. Such a work, while containing elements of fiction, is more of a chronicle; in this case, a work attempting to convey in a fictional setting the intellectual and cognitive issues confronting human beings involved in shaping the science and technology of their future. Had the book been a piece of conventional popular science writing, I would have been limited to what is known about the motives and thoughts of the people involved, while if a work of science fiction or a general novel had been my goal, then the story would have to adhere to the principles and conventions of those genres, concentrating on the development and change of word views of the book's characters through the resolution of conflicts. But in scientific fiction the overriding goal is very different. It is to present a lively and comprehensible exposition of the intellectual and emotional uncertainties involved in shaping the future of human knowledge. So in this sense, scientific fiction has as its mission to try to imagine how the world we live in today was shaped by decisions of the past, and how the decisions we take today impinge upon the world of the future (Casti, 1998: xi).

Antes de comenzar propiamente la narración, el autor autentifica a sus personajes en la ya mencionada sección "Dramatis Personae", y a continuación insiste en el carácter especulativo de la narrativa en "The Story Begins":

To separate the fact from the fantasy in these sorts of speculations, here we hypothesize that in the summer of 1949 Sir Ben Lockspeiser, Government Chief Scientist, and Sir Henry Tizard, 
science adviser to the Ministry of Defense, discussed the question of thinking machines with the celebrated novelist and physicist C.P. Snow, asking him to sound out the scientific community as to the likelihood of this 'transspeciation' coming to pass. Snow's response was to arrange an informal dinner at Christ's College, Cambridge, his alma mater, to which he invited Turing, along with several other seminal thinkers whose diverse backgrounds and interests all bore upon the general question as to whether machines could ever be built that would actually think. What follows is a speculative account of the ideas circulating around the dinner table that June evening in 1949 (Casti, 1998: xxii-xxiii).

Como puede verse, y contrariamente a lo que esperaríamos de un novelista convencional, Casti nos exhibe su plan de acción e incluso nos confiesa cómo es que ha trabajado los parlamentos: "[...] it should not come as a surprise to the reader that the fictional account of the hypothetical -but possible- gathering presented here will on occasion see the participants making statements that in ways depart from what we might imagine they would have said on the basis of their published works" (Casti, 1998: xii).

¿Qué impresión nos daría si no hiciera todas esas aclaraciones? ¿Tomaríamos la narrativa al pie de la letra, en la creencia de que la cena fue real y que sucedió y se dijo todo lo que se relata? Supóngase, por ejemplo, que no fueran Snow, Schrödinger, Wittgenstein, Turing y Haldane los personajes, sino unos comensales perfectamente desconocidos, ni siquiera científicos. ¿Podrían hablar y opinar de la misma manera sobre la inteligencia artificial? ¿Nos parecería igual de correcta la analogía "The neuron itself can be thought of as a particularly primitive kind of switch that at any moment can be only either 'ON' or 'OFF'" (Casti, 1998: 45)? Pero además de que Casti no quiere engañarnos, nos presupone lectores con cierta cultura científica; de otro modo, por qué habría de aclararnos (nuevamente en la nota de autor) lo siguiente:

For the sake of exposition, I have moved several conceptual themes in AI from their actual time in the post-1950 decades back to the period of this dinner. The reader should not infer from this that I am arguing that ideas such as Noam Chomsky's theory of language acquisition or John Searle's notorious Chinese Room argument were really developed by any of the dinner guests. It is purely a speculative issue of imagining [cursivas de Casti] that these ideas had been put forth at that time. How might the dinner-party participants have reacted? The book's final section corrects all such achronologies, and gives pointers to further reading on these and other matters discussed in the body of this narrative (Casti, 1998: xiii). 
Es muy ilustrativo comparar las advertencias de Casti con las propuestas de Carl Djerassi, eminente científico transformado en literato por decisión propia. Sus novelas pertenecen al género que él ha llamado no "ciencia ficción" sino "ciencia en ficción" (y que algunos designan "ficción científica"), ${ }^{3}$ cuyo objetivo es hacer llegar la ciencia al público no científico e incluso al anticientífico mediante esa forma literaria:

La mayoría de las personas sin educación científica se acobardan ante la ciencia. Levantan una barrera en el momento en que saben que algunos hechos científicos están por caerles encima. Es a esta porción del público a la que quiero llegar. En lugar de empezar con el agresivo preámbulo "permítanme hablarles sobre mi ciencia", prefiero dar comienzo con el más inocente "permítanme contarles una historia" y luego incorporar a la trama ciencia realista y a científicos parecidos a los de la vida real" (Djerassi, 1996).

Dicho de otra manera, en oposición a la ciencia ficción (como ya se dijo antes), el género mucho menos frecuente de la ciencia en ficción tiene como base la ciencia legítima:

Como miembro que soy de una tribu (la científica), me exijo cierto grado de exactitud y verosimilitud que impriman a mi relato un elemento importante de realidad en la ficción. Aparte de algunas concesiones inevitables que impone la narrativa, [mantengo] un alto nivel de verosimilitud [...] Salvo por alguna pequeña predatación en la cronología (de modo que se ajuste a la trama de mi novela), ningún aspecto importante [de la temática científica] es inventado. Tampoco [lo es] el comportamiento de los varios científicos protagonistas [...] Para mí, una novela sólo puede ser tratada como 'ciencia en ficción' si toda la ciencia (es decir, qué es lo que hacemos) y la mayor parte de la conducta idiosincrásica de los científicos (es decir, cómo lo hacemos) ahí descritas son verosímiles. Ninguna de estas restricciones se aplica a la ciencia ficción. Pero si uno realmente desea hacer uso de la ficción para deslizar hechos científicos en la conciencia de un público científicamente lego, entonces es crucial que los hechos que subyacen en el quehacer científico se describan con precisión. De no ser así, ¿cómo podrá el lector que carece de formación científica distinguir entre lo que se le presenta como ciencia para el entretenimiento y lo que es información? (Djerassi). ${ }^{4}$

\footnotetext{
${ }^{3}$ Guillermo Cabrera Infante acuña otra denominación al referirse a la novela de Jorge Volpi, En busca de Klingsor: “Esta obra es una muestra ejemplar del arte que quiero llamar ciencia-fusión. Fusión de la ciencia con la historia, la política y la literatura para conformar eso que llamamos cultura". Volpi, por su parte, aclara: "La escritura de un libro presupone, necesariamente, la existencia de muchos otros. Por ello, aunque ésta es una obra de ficción, considero necesario dejar constancia de algunos libros que resultaron imprescindibles para tramar su contexto histórico, sobre todo biografías científicas".

En busca de Klingsor narra la historia de un joven teniente que recibe la orden de capturar al científico que controló las investigaciones atómicas del III Reich. Klingsor, su nombre clave, supervisaba la organización secreta de físicos y místicos de la SS dedicada a resolver la construcción de la bomba atómica. Hasta donde sé, nadie la ha leído como divulgación.

${ }^{4}$ Extractos de los prefacios a Inmaculada concepción furtiva (2002), El gambito de Bourbaki (1996), La semilla de Menachem (2001), El dilema de Cantor (1997) y NO (2003).
} 
Pero así como Djerassi acepta que ha escrito novela, aunque con especiales características que apelan a la "verdad científica", Casti niega de entrada, en fina sintonía con lo que ha dicho Jurdant, que su obra pertenezca a dicho género literario. Djerassi supone haber resuelto el dilema ficción (entretenimiento)-verdad (ciencia) mediante "la descripción precisa de los hechos científicos" (que es otra manera de decir que no se los ha inventado); en tanto que Casti pretende librarlo mostrándonos sus cartas creativas desde el comienzo. Aun así, para el lector carente de información científica, ¿basta lo anterior para distinguir lo "inventado" de lo "real"? ¿Puede estar seguro de que "The uncertainty arising from the quantum-mechanical nature of the electron has nothing whatsoever to do with the problem of free will versus determinism as it pertains to human behaviour" (Casti, 1998: 20), o sólo es la opinión de Shrödinger, o es la opinión de Casti en boca del personaje?

El lector tiene ante sí un problema insoluble e injusto (tal vez hasta indetectado). Pero el divulgador consciente le ofrecerá algunos asideros, como las marcas de veracidad y las costuras evidentes.

\section{Las evidencias de veracidad}

Para no ocuparnos más de Djerassi, digamos que por el solo hecho de haber declarado que escribe novelas, leeremos (y en su momento analizaremos) sus obras como tales. Aunque la ciencia que presenta sea cuidada, precisa y legítima, esto en principio no nos incumbe como lectores de novela. Claro que su segunda intención declarada (en realidad la sustancial) es llegar al lego, pero la tomaremos en el sentido en que una novela inglesa del XIX nos deleita y a la vez, si queremos, nos instruye sobre psicología, sociología, género, historia o cualquier otra cosa. Dicho de otra manera, lo literario no se identifica con un objetivo práctico. A la literatura no le preocupa que el texto se lea como otra cosa, ni tampoco le compete el dilema amenidadveracidad; como dice Eagleton (1988) en Una introducción a la teoría literaria, el escrito nace literario pero nada puede asegurarse sobre su futuro.

La negativa de Casti a que El quinteto de Cambridge sea clasificada como novela, en cambio, nos lleva a otro tipo de consideraciones. Dicho por el propio autor, se trata de una obra de divulgación (popular science writing), pero no es convencional, porque contiene elementos de 
ficción. En este caso, el dilema cobra importancia, pues la divulgación sí tiene un objetivo práctico, una intencionalidad declarada: comunicar la ciencia al público lego. No sólo debe, por las razones que apunta Jurdant, negar su pertenencia a la literatura, sino que ha de certificar que proviene de la ciencia legítima.

La negativa consiste (como ya hemos visto) en la clasificación que el autor hace de su obra y en la serie de advertencias sobre las situaciones hipotéticas y ficticias. La "garantía del origen del texto", su credibilidad como "producto genuino de la actividad científica", se logra mediante los términos científicos utilizados y también gracias a la bibliografía comentada que ofrece en "Afterwards" (y que sirve para el lector que desee una información más amplia o profunda). Pero hay otras marcas o evidencias de veracidad que tienen relación con la "función autorreferencial" para la ciencia y la noción de que los divulgadores escriben más que nada para sus colegas científicos: la formación del escritor (como científico, datos disponibles en la solapa del libro), su pertenencia a la comunidad (miembro de prestigiadas instituciones científicas, ver la solapa) y su fama pública como divulgador (obras anteriores y alabanzas de miembros destacados de la comunidad, como consta en la $4^{\mathrm{a}}$ de forros). Todas estas marcas atestiguan su seriedad, sobre todo a los ojos de los científicos pues, como puede imaginarse, esta información es prescindible para el lego. Como ya esperábamos, la veracidad sólo puede ser juzgada por el especialista, el que domina el tema científico, pero al que menos (aparentemente) le puede interesar como lector.

Sabemos que la literatura no es una calca de la realidad; pero aun dentro de su ficcionalidad, los personajes deben ser creíbles. Casti ha elegido, por un lado, reproducirlos lo más cercanamente posible a los personajes históricos, como ya mencionamos. Pero también ha optado por una práctica muy extendida entre los divulgadores: la ruptura (deliberada o no) de la prosa literaria para intercalar la información referente a la ciencia, con la finalidad de darle el toque de seriedad necesario y de evitar en lo posible la violación al mandamiento de fidelidad a la ciencia. Dentro de este estilo didáctico-informativo de costuras a la vista (que en los textos literarios suele ser una característica indeseable), los adolescentes hablan como citando sus libros escolares, los tíos siempre son científicos y disponen de tiempo para explicar, y las amas de casa arrojan parrafadas de enciclopedia, todo intercalado con parlamentos comunes. Para 
que esto se pueda dar, el divulgador inserta oportunamente una visita a un museo, la asistencia a una conferencia, la consulta de un libro, el encuentro casual con un científico de renombre, y situaciones similares.

Veamos algunos ejemplos de costuras visibles en El quinteto de Quintet. A punto ya de terminar la sopa, cuando la discusión se ha centrado en la solución de problemas matemáticos mediante máquinas y Wittgenstein se inconforma con la idea de que una de esas máquinas pueda llegar a razonar como ser humano, Turing se dirige a sus interlocutores (quienes, no lo olvidemos, son algunas de las mentes más brillantes del siglo y además le han estado dando vueltas al mismo problema, razón por la que Snow los ha invitado):

Sucking in his breath, Turing paused for a moment, thinking back to his many readings and conversations on neurophysiology, and biological processes. Finally, he offered the group a greatly compressed and simplified account of how the brain works.

The human brain is composed of a very large number of elements called neurons, ten thousand million or more according to some people's estimates. These neurons are connected to each other through an extremely dense network of 'wires' called axons and dendrites (Casti, 1998: 45).

Poco después, los comensales discuten sobre las teorías de la adquisición del lenguaje como un proceso de simple memorización:

"Could you please briefly explain one or two of these theories, Haldane?" entreated Snow, somewhat impatient to have him get to the core issues surrounding the language acquisition problem.

"I am sure that you have all heard of the school of psychological thinking called the behaviorists, or sometimes just behaviorism," stated Haldane (Casti, 1998: 117).

Y ya para los cigarros y el brandy, refiriéndose a la cuestión del alma y a la distinción entre animales y humanos:

At this introduction of non-human animals into the discussion, Snow enquired: "You may see a centipede as having a soul, Schrödinger, but this is not really an objective claim. It strikes me more as a matter of faith or even an appeal to a sectarian religion."

"I beg to disagree," said Shrödinger apologetically. "This is not at all a religious view Eastern or otherwise. For instance, Leibniz argued in the seventeenth century that everything, man or rock, was composed of an infinite number of tiny souls. And I think Leibniz's contemporary Benedict de Spinoza would have agreed with him" (Casti, 1998: 159). 
En estos (y otros muchos) ejemplos, es obvio que el personaje en turno no se dirige a sus contertulios, sino al lector curioso que "sentirá cosquilleos por la diferencia entre lo que se supone que sabe y lo que se supone que no sabe".

Casti tiene la pericia literaria para involucrar al lector a tal grado que lo hace sentirse un invitado más, un comensal invisible asistente a la discusión en lugar de mero espectador. Dejemos claro entonces que estas costuras evidentes, que cumplen su función en la divulgación, no nos impiden exclamar: "ipero qué bien escrito está!”.

Desde el punto de vista de la divulgación de la ciencia, indudablemente que esta obra es fascinante; tal vez Stewart la llame "experimento literario" porque (como anticipa Jurdant) el autor revela desde las primeras líneas su intención, confesando incluso su manera de llevar a cabo la narrativa. Las costuras se notan, convirtiendo lo que parecía un defecto literario en una necesidad. El dilema amenidad-veracidad se ha sorteado.

Pero no hemos librado la disyuntiva literatura-ciencia.

\section{Entonces, ¿cómo leer la divulgación?}

Los dedicados a esta labor sabemos que es imposible encontrar recetas para lograr un buen texto, en especial si por consejo, moda o convicción, pretendemos que la divulgación tenga una fuerte componente literaria. Una de las cuestiones más espinosas es la de los límites de la ficción, sobre todo cuando la labor nos exige fidelidad al concepto científico y a la vez, por definición, espera de nosotros creatividad e imaginación. Nos preocupa que la comunidad científica juzgue que hemos dejado irreconocible la "ciencia legítima", y también que nuestros lectores sean incapaces de distinguir entre la "realidad" y la ficción. Estos problemas han adquirido importancia por la reciente incursión de los divulgadores en la novela como género para hacer divulgación de la ciencia.

El quinteto de Cambridge, un magnífico ejemplo de lo anterior por el interés del tema que toca y su impecable factura, nos ha servido para esbozar esa problemática, que empieza a ser abordada por analistas como Ron Curtis y Badouin Jurdant. John Casti asegura que no ha escrito novela, sino ficción científica, es decir, integra elementos de ficción y de realidad científica; por otra parte, ha utilizado el diálogo para dar cuenta de los recovecos del 
razonamiento científico. El texto resulta seductor porque no va al grano diciéndonos de manera clara y elemental lo que se sabe de la inteligencia artificial, sino que para ello utiliza múltiples recursos que pertenecen a la narrativa. Pero al hacerlo, deja establecido (como Carl Djerassi) que la ciencia de la que habla es "auténtica", y lo reitera mediante marcas, directas o indirectas, como aclaraciones sobre las licencias literarias que se tomó, o las costuras, es decir la irrupción del texto científico-didáctico en el texto narrativo. La solución de continuidad no es fácil de lograr: es verdaderamente un asunto de alta costura.

Estas enormes dificultades nos hacen pensar que quizá la novela no sea el género más adecuado, lo que a su vez nos lleva a preguntarnos cuál es la finalidad de la divulgación: ¿despertar interés, dar información, lograr un aprendizaje, causar disfrute? La solución del dilema literatura-ciencia es inalcanzable si no se considera el objetivo de cada acto de divulgación; ¿qué sentido tiene elegir el género novela si tenemos que estar poniendo a cada momento un aviso de legítimo o inventado; si recurrimos a diálogos forzados o banales para explicar un tema; si nuestros personajes son impostados científicos que todo lo saben o si la ciencia pierde terreno ante lo ameno de la mera narrativa?

Con todo, hay quienes se animan a transitar por el sendero literario sin dejar ver las preocupaciones anteriores, como auténticos artífices de alta costura. Suele ocurrir entonces que lo que resulta es simplemente literatura, independientemente de que el tema de fondo sea la ciencia; no tiene un objetivo práctico que lo limite, ni disyuntiva fidelidad-recreación. Ejemplos destacadísimo de lo antes dicho son El sistema periódico de Primo Levi, Sueños de Einstein de Alan Lightman, y Cosmicómicas de Italo Calvino. Calvino nos dice en el prólogo, a propósito de la mezcla de datos científicos e invención fantástica en sus cuentos: “Yo quisiera servirme del dato científico como de una carga propulsora para salir de los hábitos de la imaginación y vivir incluso lo cotidiano en los confines más extremos de nuestra experiencia. Cada "cosmicómica" encuentra su punto de partida en una frase leída en un libro científico allí donde una imagen logra cobrar forma y desarrollarse y vivir con una vida autónoma".

Si quiero leer ciencia legítima o explicarme algún tema, como la singularidad espaciotemporal antes de la Gran Explosión, quizá lo conveniente no sea leer Cosmicómicas, sino recurrir a libros de texto o incluso a un artículo especializado. Si dejo de lado las finalidades, 
ANA MARÍA SÁNCHEZ MORA. "EL RECURSO DE LA FICCIÓN..."

objetivos y pretensiones didáctico-informativo-formativos de la divulgación, me deja de preocupar el problema de la fidelidad, como ocurre con cualquier otra obra literaria. Claro que en este (para mí) idílico caso, y con toda razón, ya nadie me considerará divulgadora legítima. 


\section{Bibliografía}

BROCKMAN, John (1996): The Third Culture, Nueva York: Simon \& Schuster.

CALVINO, Italo (1994): Memorias del mundo y otras cosmicómicas, Madrid: Siruela.

CAREY, John (ed.) (1995): The Faber Book of Science, Londres: Faber and Faber.

CASTI, John (1998): The Cambridge Quintet, Reading: Addison-Wesley.

CURTIS, Ron (1994): “Narrative Form and Normative Force: Baconian Story-Telling in Popular Science”.

En Social Studies of Science, Núm. 24, pp. 419-61.

DJERASSI, Carl (1996): El gambito de Bourbaki, México: FCE.

- (1997): El dilema de Cantor, México: FCE.

- (2001): La semilla de Menachem, México: FCE.

- (2002): Inmaculada concepción furtiva, México: FCE.

- (2003): NO, México: FCE.

EAGLETON, Terry (1988): Una introducción a la teoría literaria, México: FCE.

EINSTEIN, Albert (1979): Relativity, Londres: Methuen.

JURDANT, Baudouin (1993): “Popularization of science as the autobiography of science". En Public Understanding of Science, Núm. 2, pp. 365-373.

KELLY, Jean et alts. (2003): "Straight/Narrative? Writing Style Changes Readers Perceptions of Story Quality". En Newspaper Research Journal, Núm. 24, pp. 4, 118-122.

LEVI, Primo (1990): El sistema periódico, México: Patria-Conaculta.

LIGHTMAN, Alan (1993): Sueños de Einstein, Barcelona: Tusquets.

LÓPEZ BELTRÁN, Carlos (2002): "Fronteras: sobre el lenguaje común y el lenguaje científico". En Antología de la divulgación de la ciencia en México, México: DGDC-UNAM.

NEGRETE, Aquiles (2002): "Science via fictional narratives: communicating science through literary forms". En Ludus Vitalis, Núm. 10, 18, pp. 197-205.

NEGRETE, Aquiles y Cecilia Lartigue (2004): “Learning from education to communicate science as a good story". En Endeavour, Núm. 28, 3, pp. 120-124.

SÁNCHEZ MORA, Ana María (2000): La divulgación de la ciencia como literatura, México: DGDC-UNAM.

VOLPI, Jorge (1999): En busca de Klingsor, México: Planeta. 